\title{
Lower critical solution temperature versus volume phase transition temperature in thermoresponsive drug delivery systems
}

\author{
M. Constantin ${ }^{1}$, M. Cristea ${ }^{1}, P$. Ascenzi $^{2}$, G. Fundueanu ${ }^{1,3^{*}}$ \\ ${ }^{1}$ Department of Bioactive and Biocompatible Polymers, 'Petru Poni' Institute of Macromolecular Chemistry, 700487 \\ Iassy, Romania \\ ${ }^{2}$ Department of Biology and Interdepartmental Laboratory for Electron Microscopy University Roma Tre, I-00146 Roma, \\ Italy \\ ${ }^{3}$ Department of Pharmaceutical Sciences, University of Ferrara, I-44100 Ferrara, Italy
}

Received 20 January 2011; accepted in revised form 28 March 2011

\begin{abstract}
One of the most subtle problem in the characterization of thermoresponsive polymers is the evaluation of the relationship between the lower critical solution temperature (LCST) of the linear polymer and the volume phase transition temperature (VPTT) of the corresponding hydrogel. Here, the LCST and the onset temperature of linear poly(N-isopropylacrylamide-co-N-hydroxymethyl acrylamide) has been determined under pseudo-physiological conditions by cloud point (CP) measurements and by microcalorimetric analysis. The LCSTs, as well as the onset temperatures, determined by the CP method, decrease with increasing the concentration of the polymer solution. On the contrary, microcalorimetric analyses give almost the same values for LCSTs and the onset temperatures regardless of polymer concentration. The VPTT of the hydrogel, determined by the blue dextran method, was found to be closely similar to the LCST of the concentrated polymer solution $(10 \%, \mathrm{w} / \mathrm{v})$, determined by the CP method. In fact, the hydrogel could be considered as a concentrated polymer solution whose concentration could be related to the amount of water retained by the hydrogel. Hydrogel microspheres have been also reported to release diclofenac, a drug model system, in a pulsating way at temperatures slightly below and above the VPTT.
\end{abstract}

Keywords: smart polymers, thermoresponsive hydrogels, lower critical solution temperature, volume phase transition temperature, cloud point

\section{Introduction}

In the last decades, much attention has been focused on thermoresponsive drug delivery systems [1-3]. Indeed, temperature variations trigger changes of the polymer configuration, leading to the modulation of the release rate of incorporated drugs. Poly(N-isopropylacrylamide) (poly(NIPAAm)) is the most relevant thermoresponsive polymer for biomedical applications, because it possesses a sharp phase transition or a lower critical solution temperature (LCST) around $32^{\circ} \mathrm{C}$ in aqueous solutions [4,
5]. Below the LCST, the polymer is in the hydrated state and therefore it is soluble. Above the LCST, the polymer is dehydrated, becomes hydrophobic, and precipitates. Accordingly, the cross-linked hydrogels obtained from this polymer swell under the critical temperature and collapse above it. The critical temperature of the hydrogel is called volume phase transition temperature (VPTT) and it is often confused with the LCST $[6,7]$. For determining the LCST of linear thermoresponsive polymer, the cloud point $(\mathrm{CP})$ method is used to determine

\footnotetext{
${ }^{*}$ Corresponding author, e-mail: ghefun@icmpp.ro
}

(c) BME-PT 
the CP value [8], and the differential scanning calorimetry analysis applies to obtain the endothermic transition peak [9].

Usually, the concentration of the polymer solution used for the determination of the LCST value by calorimetric and absorbance studies is low $(0.5-$ $1 \%, \mathrm{w} / \mathrm{v})[10,11]$. The LCST value determined at low concentration is then taken as the VPTT of the hydrogel, although the physical and chemical crosslinked hydrogels used in biomedical applications are prepared starting from high concentrated polymer solutions $(5-10 \%, \mathrm{w} / \mathrm{v})[11,12]$. Moreover, cross-linked hydrogels in the swollen state could be considered as a concentrated polymer solution related to the amount of water retained by the hydrogel.

The VPTT values of thermoresponsive hydrogels are generally determined by the equilibrium swelling ratio method [13, 14]. Hydrogels (most in the form of discs or slabs) are weighed and allowed to reach their equilibrium swelling state at different temperatures. Then, the hydrogels are taken out from the solvent and the excess of solvent present on their surface is removed by blotting with filter paper. However, this method, that allows to determine the water retained at different temperatures, is unreliable since the gel is taken out at room temperature and tapped for a random time with a filter paper or laboratory tissue $[15,16]$. Moreover, in the case of microspheres, this method gives wrong results, since it is not possible to discriminate between the water that is present in the microspheres and the water present between the microspheres.

On the basis of these considerations, the main purpose of this paper is to evaluate the correspondence between the LCST values of the polymer solutions at different concentrations and the VPTT values of the corresponding hydrogels. Firstly, we synthesized the poly(N-isopropylacrylamide-co-N-hydroxymethyl acrylamide) (poly(NIPAAm-co-HMAAm)) copolymer with a LCST value (under physiological conditions) closely similar to the body temperature. Secondly, we studied the influence of the solution concentration on the LCST value by DSC and CP methods. Thirdly, the linear thermoresponsive polymer was transformed in cross-linked hydrogel microspheres, and the VPTT was determined by the blue dextran method and compared with the LCST value. Finally, the release of diclofenac, a drug model system, at temperatures situated slightly below and above the VPTT was investigated.

\section{Materials and methods \\ 2.1. Materials}

$\mathrm{N}$-isopropylacrylamide (NIPAAm) (from SigmaAldrich Chemical Co., Milwaukee, WI, USA) was re-crystallized with hexane. The N-hydroxymethylacrylamide (HMAAm) aqueous solution (48 wt \%), the glutaraldehyde (GA) aqueous solution $(25 \%$, w/v), 1,4-dioxane, and N,N'-azobisisobutyronitrile (AIBN) were supplied from Fluka AG (Buchs, Switzerland). 1,4-Dioxane was purified by refluxing and AIBN was purified in methanol. Light mineral oil $\left(d=0.84 \mathrm{~g} \cdot \mathrm{ml}^{-1}\right)$ and diclofenac ( $\mathrm{Na}$ form) were purchased from Sigma-Aldrich Chemical Co. (St Louis, MO, USA). Blue dextran (BD) was provided from Pharmacia (Uppsala, Sweden). All the other chemicals were from Fluka AG (Buchs, Switzerland). All chemicals were of the highest analytical grade.

\subsection{Synthesis of poly(NIPAAm-co-HMAAm)}

Synthesis of linear poly(NIPAAm-co-HMAAm)) was carried out by free radical copolymerization in 1,4-dioxane with AIBN as the initiator. Typically, $1.13 \mathrm{~g}$ of NIPAAm (10 mmol), $0.4 \mathrm{ml}$ of HMAAm $(2 \mathrm{mmol})$ and $0.010 \mathrm{~g}$ AIBN were solubilized in $12 \mathrm{ml}$ of 1,4-dioxane. Dried nitrogen was bubbled through the solution for $30 \mathrm{~min}$. prior to polymerization. The reaction mixture was allowed to react at $70^{\circ} \mathrm{C}$ for 16 hours. The polymer was precipitated into diethyl ether, and dried under vacuum. Finally, the copolymer was solubilized in distilled water, dialyzed for 7 days at $22^{\circ} \mathrm{C}$ (molecular weight cut off $10000-12,000 \mathrm{Da}$; from Medi Cell International, England), and recovered by freeze-drying.

\subsection{Copolymer composition}

The copolymer composition was determined by ${ }^{1} \mathrm{H}$ NMR analysis. ${ }^{1} \mathrm{H}$ NMR spectra of poly (NIPAAm-co-HMAAm) were recorded in $\mathrm{D}_{2} \mathrm{O}$ on a Varian Mercury Plus 400/Varian VXR 200 spectrometer operating at the frequency of $400 \mathrm{MHz}$. The molar fraction of HMAAm in poly(NIPAAmco-HMAAm) was calculated according to Equation (1): 


$$
\begin{aligned}
3 \text { HMAAm } & =(3 \text { HMAAm }+3 \text { NIPAAm }+ \\
& +6 \text { NIPAAm })-Y \cdot 9 \text { NIPAAm }
\end{aligned}
$$

where $Y$ is the molar fraction of NIPAAm calculated as the area of the methynic proton at $3.91 \mathrm{ppm}$, and (3 MAAm + 3 NIPAAm + 6 NIPAAm) is the total area of the peaks between 0.5 and $2.5 \mathrm{ppm}$, corresponding to the main backbone protons (3 HMAAm +3 NIPAAm) and the NIPAAm methylenic protons (6 NIPAAm).

\subsection{Determination of the molecular weight of poly(NIPAAm-co-NHMAAm)}

The number-average $\left(M_{\mathrm{n}}\right)$ and weight-average $\left(M_{\mathrm{w}}\right)$ molecular weight of poly(NIPAAm-co-HMAAm) were determined by GPC using an instrument GPCPL-EMD 950 from Polymer Laboratories (Shropshire, UK) (in dimethylformamide at $120^{\circ} \mathrm{C}$, the flow rate was $0.7 \mathrm{ml} / \mathrm{min}$ ). Calibration was carried out with monodisperse polystyrene standards.

\subsection{LCST determination}

LCST was determined from the absorbance at $450 \mathrm{~nm}$ measured against temperature using an UV-Vis spectrophotometer coupled with a temperature controller. The polymer solution was prepared in standard phosphate buffer $(\mathrm{pH}=7.4,50 \mathrm{mM}$ $\mathrm{Na}_{2} \mathrm{HPO}_{4}$ and $\mathrm{NaOH}$ ) (PB). The heating rate was $0.2^{\circ} \mathrm{C}$ every 10 minutes. $\mathrm{CP}$ was defined as the temperature corresponding to the absorbance value of 0.5 of the curve of the absorbance versus temperature. The onset temperature was defined as the temperature at which the first signs of turbidity occurred.

Microcalorimetric analyses were performed in PB with a DSC III microcalorimeter (Setaram, Caluire, France) at a heating rate of $0.5^{\circ} \mathrm{C} / \mathrm{min}$, under nitrogen. The transition temperature was defined as the temperature corresponding to the peak of the thermogram. The onset temperature was determined from crossing of the baseline with the leading edge of the endotherm.

\subsection{Microsphere preparation}

Typically, $1 \mathrm{~g}$ copolymer was solubilized in $10 \mathrm{ml}$ distilled water, at $22^{\circ} \mathrm{C}$. Then, $0.4 \mathrm{ml}$ of $0.5 \mathrm{M}$ $\mathrm{H}_{2} \mathrm{SO}_{4}$ and $0.5 \mathrm{ml}$ of $25 \% \mathrm{GA}(\mathrm{w} / \mathrm{v})$ were added just before dispersion of the aqueous copolymer solution in the dispersion phase. The continuous phase consists in $100 \mathrm{ml}$ light mineral oil containing $0.5 \mathrm{~g}$ soybean lecitin, as the dispersing agent. The mixture was stirred at $350 \mathrm{rpm}$ by a three-blade turbine impeller. The reaction temperature was fixed below the LCST of the polymer solution $\left(34^{\circ} \mathrm{C}\right)$; the reaction time was 24 hours. Finally, cross-linked microspheres were washed successively with diethyl ether, methanol, water, methanol, and dried diethyl ether.

\subsection{Morphological and dimensional analysis of the microspheres}

The morphology of the microspheres was evaluated by scanning electron microscopy (SEM) (ESEM, type Quanta 200, Netherlands) and by optical microscopy using an inverted microscope (Nikon Diaphot, Tokyo, Japan) equipped with a digital camera. The mean diameter of the microspheres was determined on digital photomicrographs, considering at least 100 microspheres for each sample. The analysis was performed by the computerized size analysis system NIH image.

\subsection{Volume phase transition temperature}

The volume phase transition temperature (VPTT) corresponded to the inflexion point of the curve of $\mathrm{PB}$ retention as a function of temperature. The $\mathrm{PB}$ retention values at different temperatures were determined by the BD approach based on the solute exclusion technique at equilibrium [17]. In a closed vial, $200 \mathrm{mg}$ of dried microspheres were soaked in $10 \mathrm{ml}$ of PB containing BD $(2 \mathrm{mg} / \mathrm{ml})$. After reaching equilibrium ( 24 hours), $0.5 \mathrm{ml}$ of the solution were picked up and the BD content was assayed by UV-Vis spectrophotometry, at $618 \mathrm{~nm}$. The PB retention $\left(\mathrm{PB}_{\mathrm{R}}\right)$ values were determined according to Equation (2):

$\mathrm{PB}_{\mathrm{R}}\left[\mathrm{ml} \cdot \mathrm{g}^{-1}\right]=V_{\mathrm{i}}\left(C_{\mathrm{e}}-C_{\mathrm{i}}\right) \cdot \frac{5}{C_{\mathrm{e}}}$

where $V_{\mathrm{i}}$ is the initial volume of the BD solution [ml], $C_{\mathrm{i}}$ is the initial concentration of $\mathrm{BD}[\mathrm{mg} / \mathrm{ml}$, and $C_{\mathrm{e}}$ is the $\mathrm{BD}$ concentration at equilibrium, in the presence of microspheres $[\mathrm{mg} / \mathrm{ml}]$.

\subsection{Drug loading}

Drug-loaded microspheres were prepared by soaking $100 \mathrm{mg}$ dried microspheres in $4 \mathrm{ml}$ of ethanol containing diclofenac $(2.5 \mathrm{mg} / \mathrm{ml})$. After the complete evaporation of the solvent at $22 \pm 2^{\circ} \mathrm{C}$, the 
microspheres were washed with $10 \mathrm{ml}$ of diethyl ether and dried under vacuum at room temperature. The amount of the retained drug, per mg of dried microspheres, was determined by solubilization of the entrapped drug in ethanol. The amount of the drug in the filtrate was determined after microsphere isolation by UV-Vis spectroscopy, using a previously made calibration curve.

\subsection{In vitro drug release kinetics}

In vitro drug release kinetic studies were performed (under thermal cycling operation), at different temperatures, by soaking an appropriate amount of loaded microspheres in $50 \mathrm{ml}$ of PB solution under gentle stirring. At regular time intervals, aliquots of the released medium were withdrawn and the drug content was determined spectrophotometrically. The same volume of the released medium was added to replace the volume of the extracted samples.

\section{Results and discussion}

\subsection{Preparation and characterization of the thermoresponsive copolymer}

The biomedical applications of thermoresponsive polymers are achievable when the polymers possess a phase transition corresponding approximately to the human body temperature. Moreover, the ther- mal transition should be sharp when very small variations of temperature occur below and above the LCST. As it is well known, poly(NIPAAm) exhibits a LCST value at $32^{\circ} \mathrm{C}$ in aqueous solution $[4,5]$. Under pseudo-physiological conditions (PB at $\mathrm{pH}$ 7.4) the LCST decreases to lower temperatures [12]. In order to increase the LCST towards body temperature, NIPAAm is usually copolymerized with hydrophilic monomers [18, 19]. Here, HMAAm was selected to be copolymerized with NIPAAm. Note that the presence of HMAAm in the copolymer does not affect the acrylamide sequence of NIPAAM, which is responsible for thermorespon-

Table 1. Effect of the co-monomer ratio in the feed and in the copolymer on the LCST determined by the CP method under pseudo-physiological conditions ( $\mathrm{PB}$ at $\mathrm{pH}$ 7.4). The concentration of the copolymer was $10 \%$, w/v

\begin{tabular}{|c|c|c|c|c|c|}
\hline \multirow{3}{*}{ Sample } & \multicolumn{4}{|c|}{ Co-monomer composition } & \multirow{3}{*}{$\begin{array}{c}\text { LCST } \\
{\left[{ }^{\circ} \mathrm{C}\right]} \\
\text { In PB }\end{array}$} \\
\hline & \multicolumn{2}{|c|}{$\begin{array}{c}\text { In the feed } \cdot 10^{-3} \mathrm{M} \\
{[\% \text { molar ratio] }}\end{array}$} & \multicolumn{2}{|c|}{$\begin{array}{c}\text { In the co-polymer } \\
\text { [\% molar ratio] }\end{array}$} & \\
\hline & NIPAAm & HMAAm & NIPAAm & HMAAm & \\
\hline $\mathrm{TP}_{0}$ & 10 & 0.0 & 100 & 0.0 & $28.8 \pm 0.1$ \\
\hline $\mathrm{TP}_{1}$ & 10 & 1.0 & n.d. ${ }^{\mathrm{a}}$ & n.d. & $32.1 \pm 0.2$ \\
\hline $\mathrm{TP}_{2}$ & $10(86.96)$ & $1.5(13.04)$ & 86.18 & 13.82 & $34.5 \pm 0.2$ \\
\hline $\mathrm{TP}_{3}{ }^{\mathrm{b}}$ & $10(83.34)$ & $2.0(16.66)$ & 82.87 & 17.13 & $36.8 \pm 0.2$ \\
\hline
\end{tabular}

Data are the results of three independent experiments.

${ }^{\mathrm{a}}$ n.d. $=$ not determined

${ }^{\mathrm{b}} M_{\mathrm{n}}=21200, M_{\mathrm{w}}=76400, \mathrm{IP}=3.60, \mathrm{IP}=$ index of polydispersity

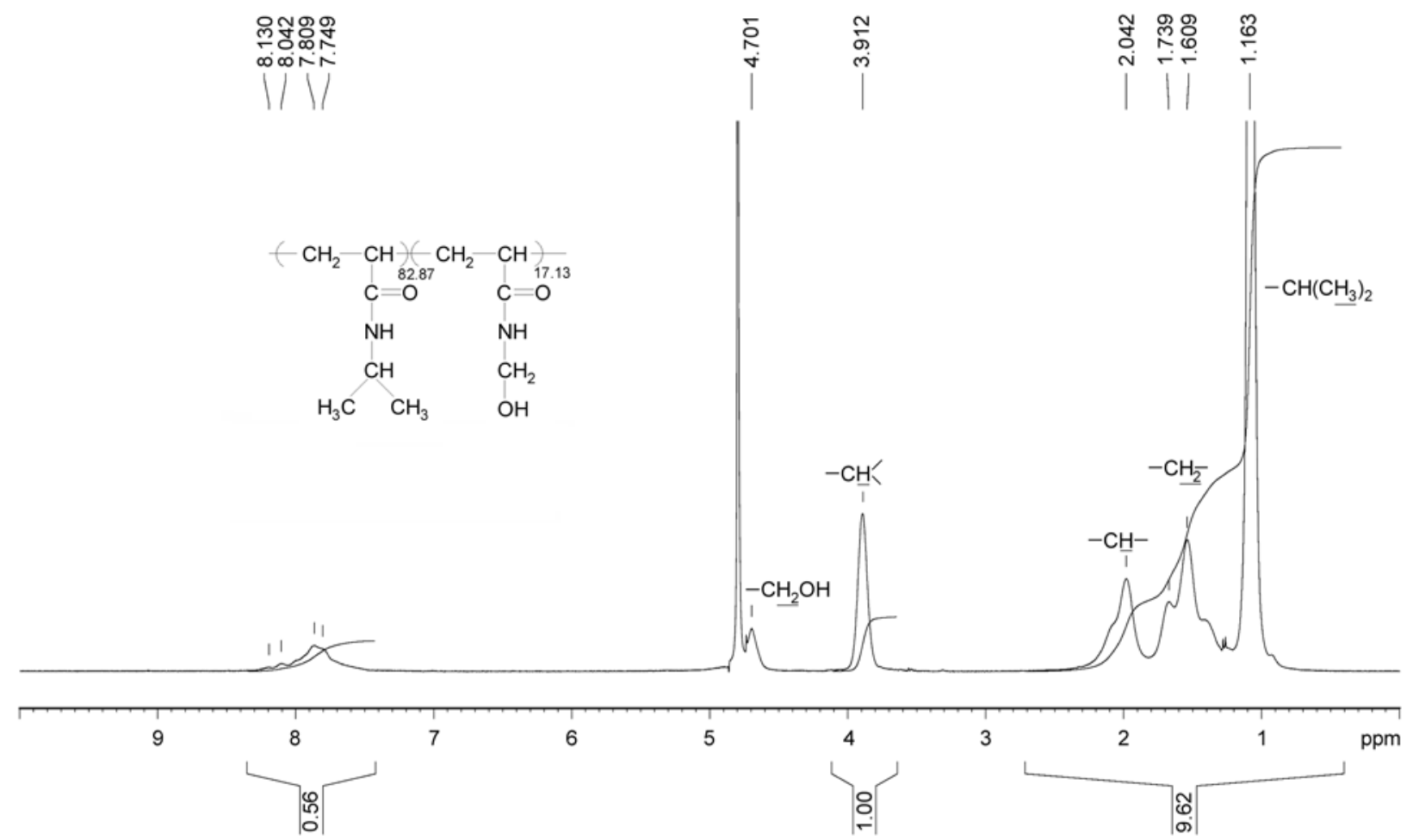

Figure 1. ${ }^{1} \mathrm{H}$ NMR spectrum of poly(NIPAAm-co-HMAAm) (sample $\mathrm{TP}_{3}$ in Table 1) 
sibility. In addition, HMAAm possesses hydroxyl groups which undergo easily to cross-linking.

As reported in Table 1 and proven by ${ }^{1} \mathrm{H}$ NMR spectra (Figure 1), the copolymer formation and the percentage of the co-monomers in the copolymer correspond approximately to those observed in the feed. It should be noted that increasing the HMAAm content of the copolymer, the LCST value increases, however it shows a sharp phase transition. The copolymer containing an initial 10/2 NIPAAm/ HMAAm co-monomer molar ratio (sample TP3) possesses a LCST value of $36.8^{\circ} \mathrm{C}$ corresponding to the human body temperature.

\subsection{Influence of the polymer concentration on LCST}

Generally, the LCST value of the hydrogels is determined by the CP method at low polymer concentration $(0.5-1 \%)[10,11]$, this value is then assumed to correspond to that of the hydrogels. However, the chemically cross-linked hydrogels are prepared from highly concentrated polymer solution $(5-10 \%)$ [11, 12]. Moreover, hydrogels could be considered as concentrated polymer solutions whose concentration could correspond approximately to the amount of the retained water. Therefore, from both the theoretical and practical viewpoints, the influence of the concentration of the polymer solution on the LCST value under pseudophysiological conditions must be determined. As shown in Table 2, the LCST value of linear poly (NIPAAm-co-HMAAm) determined by CP measurements decreases as the concentration of the polymer increases.

However, in a highly concentrated solution, the solution opalescence is more accentuated than in a diluted solution. Since this discrepancy could affect

Table 2. Effect of the polymer concentration on the LCST as determined by the $\mathrm{CP}$ method $^{\mathrm{a}}$

\begin{tabular}{|l|c|c|c|c|c|c|}
\hline \multirow{3}{*}{ Sample } & \multicolumn{6}{|c|}{ LCST $_{\left[{ }^{\mathbf{}} \mathbf{C}\right] \text { at different copolymer concentration }}^{[\%, \mathbf{w}]}$} \\
\cline { 2 - 7 } & $\mathbf{0 . 2 5}$ & $\mathbf{0 . 5}$ & $\mathbf{1}$ & $\mathbf{3}$ & $\mathbf{5}$ & $\mathbf{1 0}$ \\
\hline $\mathrm{TP}_{0}$ & 30.8 & 30.6 & 30.3 & 29.5 & 29.1 & 28.8 \\
\hline $\mathrm{TP}_{2}$ & 36.8 & 36.5 & 36.1 & 35.4 & 35.1 & 34.5 \\
\hline \multirow{2}{*}{$\mathrm{TP}_{3}$} & 39.4 & 38.6 & 38.1 & 37.7 & 37.3 & 36.8 \\
& (n.d.) $^{\mathrm{b}}$ & $(40.3)^{\mathrm{c}}$ & $(40.25)$ & $(39.9)$ & (n.d.) & $(39.66)$ \\
\hline
\end{tabular}

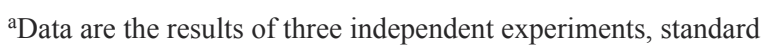
deviation $\pm 0.15^{\circ} \mathrm{C}$

$b_{\text {n.d., not determined }}$

${ }^{\mathrm{c}}$ Data between brackets were determined by the calorimetric method the exact determination of LCST, we considered also the onset points which correspond to the first opaqueness that appeared in the solution (Table 3). In addition, the influence of polymer concentration on LCST was determined by the DSC technique (Figure 2).

The onset temperature determined by the $\mathrm{CP}$ method decreases on increasing the polymer concentration. In contrast, the LCST values and the onset points determined by the calorimetric method seem to be unaffected by the polymer concentration (see Tables 2 and 3). In fact, the calorimetric technique gives information on the heat released from the cleavage of hydrogen bonds between the water solvent and the polymer [20]. Therefore, it is expected that dehydration occurs at the same temperatures regardless of the polymer concentration, while the CP method detects the clouding of the solution due to the hydrophobic interactions between the polymer chains [21]. Obviously, hydrophobic interac-

Table 3. Effect of the polymer concentration on the onset temperature as determined by the CP method ${ }^{\mathrm{a}}$

\begin{tabular}{|c|c|c|c|c|c|c|}
\hline \multirow{2}{*}{ Sample } & \multicolumn{6}{|c|}{$\begin{array}{c}\text { Onset temperature }\left[{ }^{\circ} \mathrm{C}\right] \text { at different copolymer } \\
\text { concentration }[\%, w / v]\end{array}$} \\
\hline & 0.25 & 0.5 & 1 & 3 & 5 & 10 \\
\hline $\mathrm{TP}_{0}$ & 30.7 & 30.3 & 29.9 & 29.3 & 28.9 & 28.6 \\
\hline $\mathrm{TP}_{2}$ & 36.3 & 36.1 & 35.6 & 35.1 & 34.7 & 33.9 \\
\hline $\mathrm{TP}_{3}$ & $\begin{array}{c}38.0 \\
(\text { (n.d. })^{b}\end{array}$ & $\begin{array}{c}37.8 \\
(35.75)^{\mathrm{c}}\end{array}$ & $\begin{array}{c}37.6 \\
(36.3)\end{array}$ & $\begin{array}{c}37.2 \\
(35.8)\end{array}$ & $\begin{array}{l}36.8 \\
\text { (n.d.) }\end{array}$ & $\begin{array}{c}36.5 \\
(35.94)\end{array}$ \\
\hline
\end{tabular}

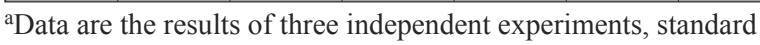
deviation $\pm 0.15^{\circ} \mathrm{C}$

${ }^{b}$ n.d., not determined

${ }^{\mathrm{c}}$ Data between brackets were determined by the calorimetric method

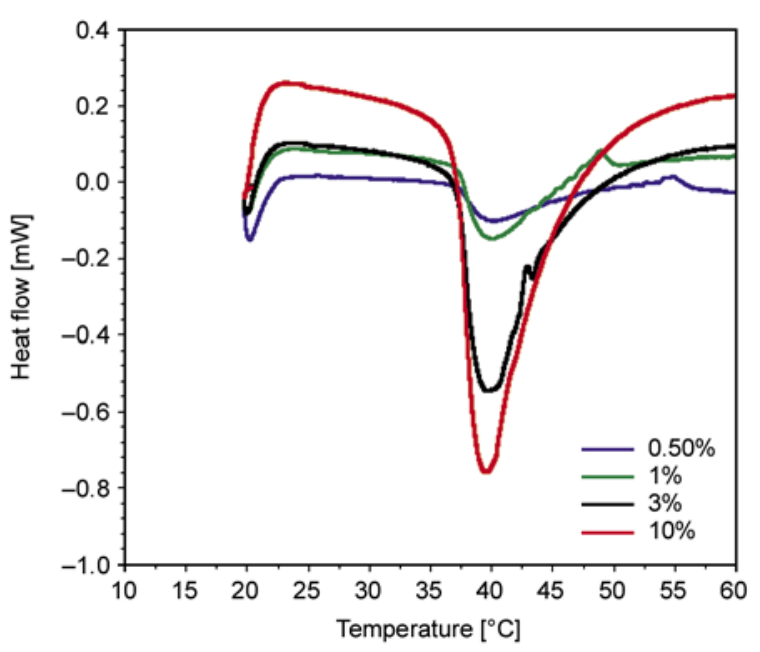

Figure 2. DSC thermograms of the poly(NIPAAm-coHMAAm) solution (sample $\mathrm{TP}_{3}$ in Table 1) in phosphate buffer at $\mathrm{pH} 7.4$ at different concentrations. The scanning rate was $0.5^{\circ} \mathrm{C} \cdot \mathrm{min}^{-1}$ 

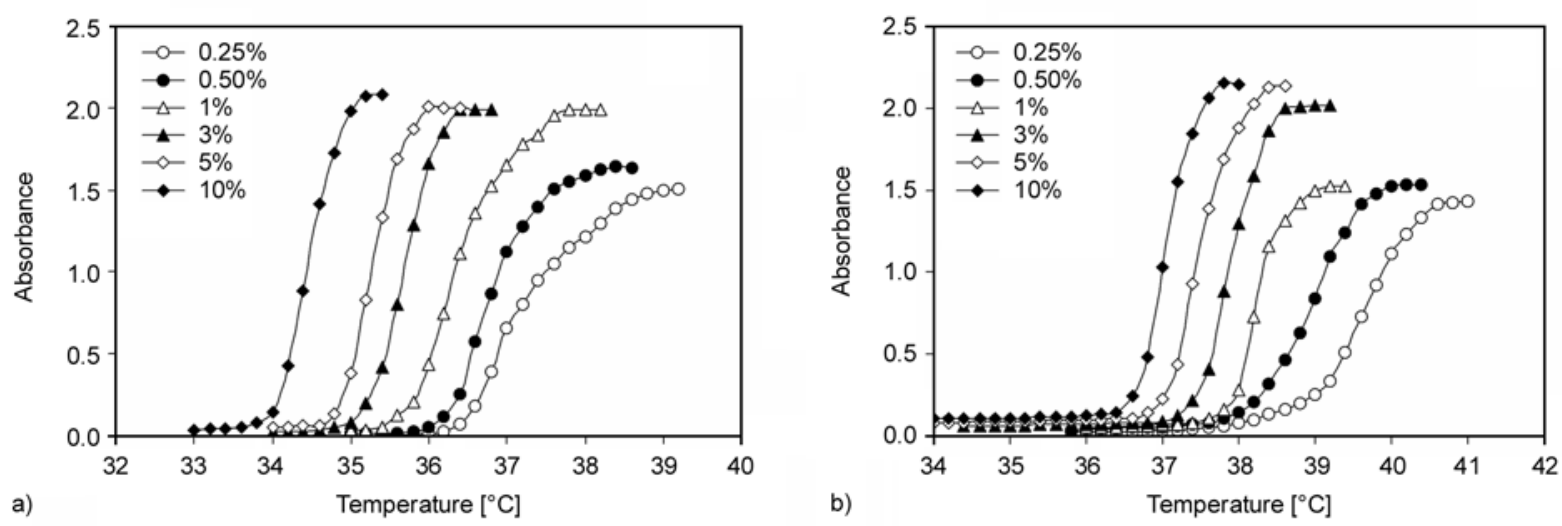

Figure 3. LCST profile of poly(NIPAAm-co-HMAAm) (sample $\mathrm{TP}_{2}$, Panel a; sample $\mathrm{TP}_{3}$, Panel b) dissolved in $\mathrm{PB}$ ( $\mathrm{pH} 7.4$ ), at different polymer concentrations $(0.25 \%$, open circles; $0.5 \%$ filled circles; $1 \%$, open triangles; $3 \%$, filled triangles; $5 \%$, open diamonds, and $10 \%$, filled diamonds)

tions are faster in a highly concentrated solution, and therefore the phase transition appears to be sharper at high polymer concentration (Figure $3 a, b$ ). In conclusion, the $\mathrm{CP}$ method is a more reliable technique since highlights the dehydration and the hydrophobic interactions of the polymer chains, giving information at the macroscopic level.

\subsection{Preparation and characterization of thermoresponsive microspheres}

Spherical poly(NIPAAm-co-HMAAm) microparticles with a mean diameter of $102 \mu \mathrm{m}$ (Figure 4a) were prepared from preformed polymers $\left(\mathrm{TP}_{3}\right)$ by cross-linking the $-\mathrm{OH}$ groups of HMAAm with GA, under acidic conditions [22].

Microspheres were prepared at a temperature as high as possible, but lower than the LCST. Preliminarily, the value of the LCST of the polymer solution $\left(=38.5^{\circ}\right)$ was determined by $\mathrm{CP}$ method under the same preparation conditions (i.e. in the presence of $\mathrm{H}_{2} \mathrm{SO}_{4}$ and GA). After cross-linking at a temperature below the $\operatorname{LCST}\left(=34^{\circ} \mathrm{C}\right)$, the microspheres still retain the thermoresponsive properties of the linear copolymer (Figure 4 b, c).

However, the most important question rising here concerns the coincidence of the VPTT value of the microspheres with the LCST value of the linear polymer. The VPTT of the hydrogel was determined by measuring the amount of PB retention at different temperatures. The PB retention of the microspheres was determined by an accurate method based on solute exclusion at equilibrium [17]. This method is based on measuring the increase of the BD concentration in supernatant, which occurs when dried microspheres are added to a solution of $\mathrm{BD}$. Therefore, the temperature dependence of the $\mathrm{PB}$ retention was monitored (Figure 5). A decrease of the PB retention was observed even at temperatures lower than those of the onset points. This behavior is somewhat unexpected since no influence of temperature on the dehydration process was previously reported for the linear polymer under peak onset values when determined by calorimetric studies (Figure 2).

Nevertheless, the decrease of the PB retention profile is discontinuous around $36.5^{\circ} \mathrm{C}$, corresponding to the VPTT of the hydrogel microspheres. This value is closely similar to the LCST value of the linear polymer at the polymer concentration of $10 \%(\mathrm{w} / \mathrm{v})$ as determined by the $\mathrm{CP}$ method (sample $\mathrm{TP}_{3}$ in Table 2). Note that the volume of $\mathrm{PB}$ retained by the hydrogel microspheres at $22^{\circ} \mathrm{C}$ is $11.2 \mathrm{ml} / \mathrm{g}$, and corresponds approximately to the concentration of the polymer solution that displays the LCST value of $36.8^{\circ} \mathrm{C}$. In conclusion, the VPTT of the hydrogel is in agreement with the LCST value of the polymer solution at the concentration corresponding to the amount of PB retained by the hydrogel.

Conflicting data are reported in the literature concerning the relationships between the phase transition of the linear polymers and the hydrogels. Some authors report that both the transition temperature and the heat of collapse of the poly(NIPAAm) gel show similar values to those of the poly(NIPAAm) solutions [23]. Other authors report that the transition temperature of poly(NIPAAm) gels is slightly higher $\left(1-2^{\circ} \mathrm{C}\right)$ than that of the linear polymer solutions $[24,25]$. This difference in the transition tem- 


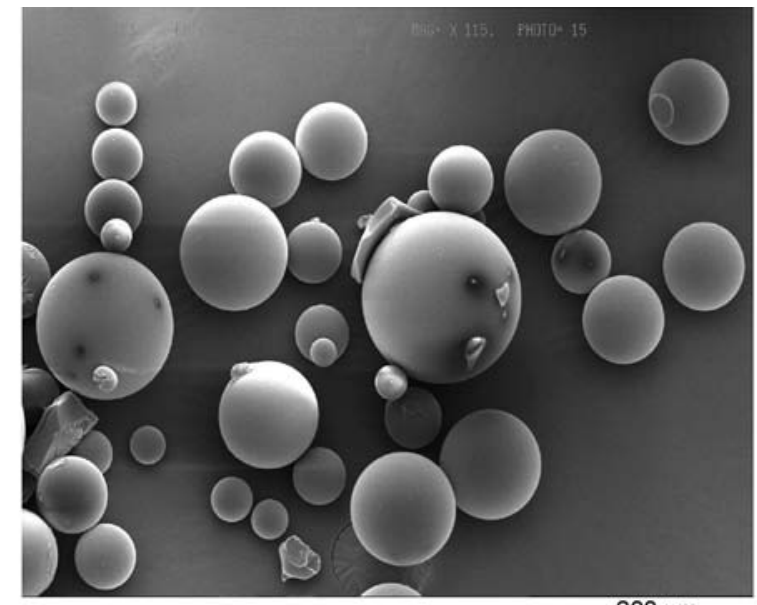

a)

$200 \mu \mathrm{m}$

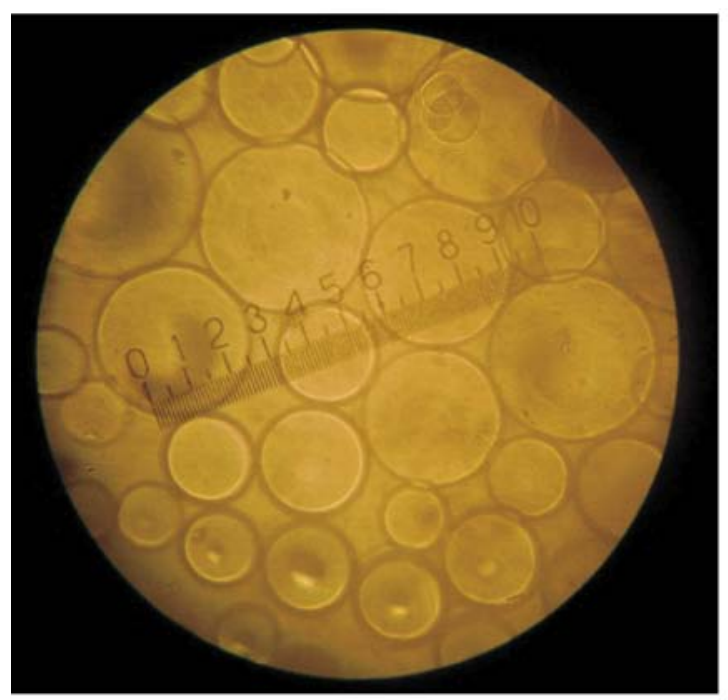

b)

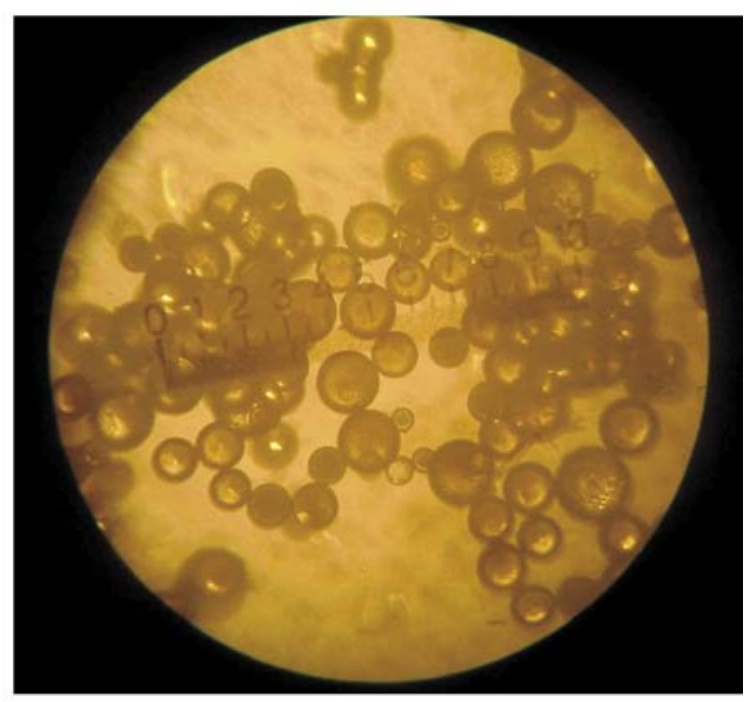

c)

Figure 4. Scanning electron micrographs of poly(NIPAAm-co-HMAAm) microspheres (Panel a). Optical photomicrographs of thermoresponsive microspheres taken in the swollen state in water below the VPTT value at $35^{\circ} \mathrm{C}$ (Panel b) and above the VPTT value at $38^{\circ} \mathrm{C}$ (Panel c). The bar corresponds to $200 \mu \mathrm{m}$ in Panel a, and $500 \mu \mathrm{m}$ in Panels b and c.

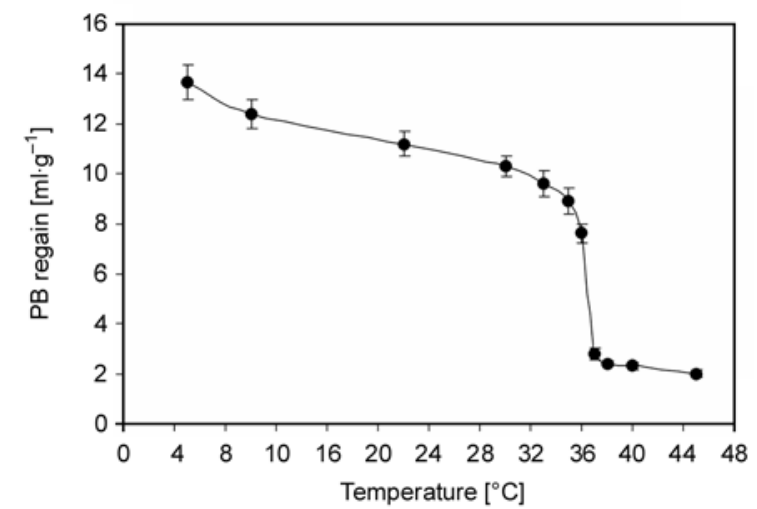

Figure 5. Effect of temperature on the PB retention by cross-linked thermoresponsive microspheres under physiological conditions. All values are the mean of three independent measurements $\pm \mathrm{SD}$. perature could be explained by the reduced 'mobility' of the polymer chains in a cross-linked network. We agree with the last interpretation; however, when the cross-linking degree is enough low, its influence is negligible.

\subsection{Drug loading and release studies}

The release of drugs by thermal cycling was performed for most of thermoresponsive hydrogels at temperatures far below and above the LCST of the linear polymer (i.e., 25 and $40^{\circ} \mathrm{C}$ ) [26, 27]. The reason is to be sure that these temperatures are also situated below and above the VPTT of the hydrogel, which in fact, is not known. However, biomedical applications of the hydrogels are only possible when 
the volume changes take place at temperatures slightly higher and lower than the VPTT. On the basis of these considerations, we studied the release of diclofenac from thermoresponsive microspheres at 35 and $38^{\circ} \mathrm{C}$, i.e. at temperatures closely near the VPTT $\left(=36.5^{\circ} \mathrm{C}\right)$ (Figure 6).

Previously, diclofenac, used as low molecular weight model drug, was loaded in thermoresponsive microspheres by the solvent evaporation method. This process was performed in ethanol since diclofenac displays a good solubility in this solvent and the thermoresponsive microspheres possess a high swelling degree in ethanol (swollen volume/dried volume $=17.9$ ). Following this procedure, a high encapsulation efficiency $(95.4 \pm 3 \%)$ was obtained, in fact almost the entire amount of the drug from solution is forced to diffuse into the microspheres during ethanol evaporation. The entrapped drug is molecularly dispersed in the network of the microspheres as proved by the absence of any fusion peak of the drug crystals in the DSC thermogram of loaded microspheres (data not shown). The release rate of diclofenac is mainly determined by diffusion of the drug through the pores of the microsphere matrix. Below the VPTT value (i.e., at $35^{\circ} \mathrm{C}$ ), the hydrogel microspheres are in the swollen state and no steric interaction between the drug and the microsphere matrix takes place; therefore, the release rate of the drug is high. On the contrary, when the temperature is raised above the VPTT (i.e., at $38^{\circ} \mathrm{C}$ ), the microsphere matrix is in a collapsed state and the diffusion of the drug is impaired. Moreover, addi-

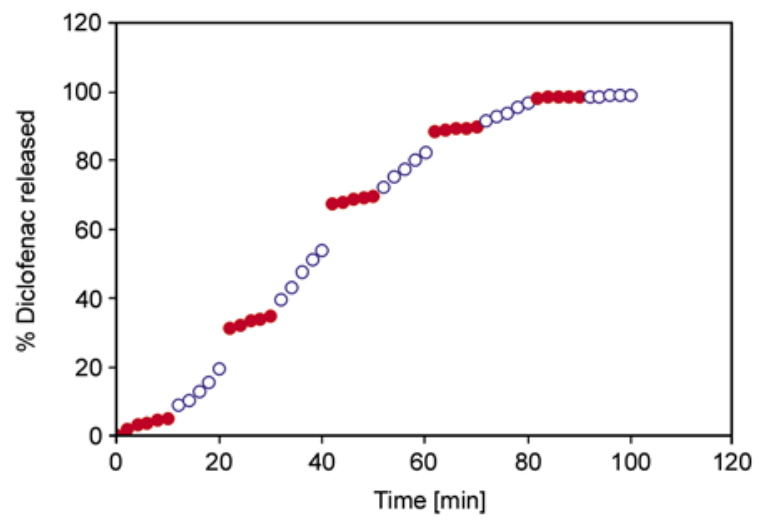

Figure 6. Effect of temperature cycling $\left(35^{\circ} \mathrm{C}(\circ)\right.$ and $\left.38^{\circ} \mathrm{C}(\bullet)\right)$ on diclofenac release from poly (NIPAAm-co-HMAAm) microspheres in phosphate buffer at $\mathrm{pH} 7.4$ tional hydrophobic interactions between the drug and the polymer network occur; accordingly, the release rate is dramatically reduced. Finally, a pulsating drug release was achieved under thermal cycling between 35 and $38^{\circ} \mathrm{C}$.

Notably, a fraction of the drug is expelled during the shrinkage of the microsphere matrix, when the temperature increases above the VPTT. This suggests the presence of the dissolved drug in the swollen hydrogel. Such drug fraction is mechanically squeezed out from the system when the hydrogel network collapsed.

\section{Conclusions}

Poly(NIPAAm-co-HMAAm)) was synthesized both as a thermoresponsive linear polymer and as cross-linked hydrogel microspheres. The LCST value, determined by the $\mathrm{CP}$ method, strongly depends to the polymer solution concentration. On the contrary, the LCST value determined by the calorimetric method is unaffected by the polymer concentration. The CP method was found to be a more reliable technique to characterize a hydrogel because takes in consideration both the dehydration and hydrophobic interactions of polymer chains, giving information at macroscopic level. The VPTT value of the hydrogel agrees with the LCST value of the polymer solution at the concentration corresponding to that of PB retained by the hydrogel. The pulsating release of diclofenac under thermal cycling operation at temperatures slightly below and above the VPTT, here determined, confirmed that the phase transition temperature of the hydrogel is well determined.

Abbreviations
\begin{tabular}{|l|l|}
\hline AIBN & N,N'-azobisisobutyronitrile \\
\hline BD & blue dextran \\
\hline CP & cloud point \\
\hline GA & glutaraldehyde \\
\hline HMAAm & N-hydroxymethylacrylamide \\
\hline LCST & lower critical solution temperature \\
\hline NIPAAm & N-isopropylacrylamide \\
\hline PB & phosphate buffer \\
\hline poly(NIPAAm) & poly(N-isopropylacrylamide) \\
\hline poly(NIPAAm-co- & $\begin{array}{l}\text { poly(N-isopropylacrylamide-co-N- } \\
\text { hydroxymethyl acrylamide })\end{array}$ \\
HMAAm & scanning electron microscopy \\
\hline SEM & volume phase transition temperature \\
\hline VPTT &
\end{tabular}




\section{Acknowledgements}

This work was supported by CNCSIS -UEFISCSU, project number 644/19.01.2009, PNII - IDEI code 989/2008. M. Constantin acknowledges the financial support of European Social Fund - 'Cristofor I. Simionescu' Postdoctoral Fellowship Programme (ID POSDRU/89/1.5/S/55216), Sectoral Operational Programme Human Resources Development $2007-2013$.

\section{References}

[1] Bae Y. H., Okano T., Hsu R., Kim S. W.: Thermo-sensitive polymers as on-off switches for drug release. Die Makromolekulare Chemie, Rapid Communications, 8, 481-485 (1987).

DOI: $10.1002 /$ marc.1987.030081002

[2] Jeong B., Bae Y. H., Lee D. S., Kim S. W.: Biodegradable block copolymers as injectable drug-delivery systems. Nature, 388, 860-862 (1997).

DOI: $10.1038 / 42218$

[3] Chearúil F. N., Corrigan O. I.: Thermosensitivity and release from poly $\mathrm{N}$-isopropylacrylamide-polylactide copolymers. International Journal of Pharmaceutics, 366, 21-30 (2009).

DOI: 10.1016/j.ijpharm.2008.08.036

[4] Boutris C., Chatzi E. G., Kiparissides C.: Characterization of the LCST behaviour of aqueous poly(N-isopropylacrylamide) solutions by thermal and cloud point techniques. Polymer, 38, 2567-2570 (1997).

DOI: $10.1016 / \mathrm{S} 0032-3861(97) 01024-0$

[5] Costa R. O. R., Freitas R. F. S.: Phase behavior of poly ( $N$-isopropylacrylamide) in binary aqueous solutions. Polymer, 43, 5879-5885 (2002).

DOI: 10.1016/S0032-3861(02)00507-4

[6] Zhuo R-X., Li W.: Preparation and characterization of macroporous poly $(N$-isopropylacrylamide) hydrogels for the controlled release of proteins. Journal of Polymer Science Part A: Polymer Chemistry, 41, 152-159 (2003).

DOI: $10.1002 /$ pola. 10570

[7] Zhao Z., Li Z., Xia Q., Xi H., Lin Y.: Fast synthesis of temperature-sensitive PNIPAAm hydrogels by microwave irradiation. European Polymer Journal, 44, 12171224 (2008).

DOI: 10.1016/j.eurpolymj.2008.01.014

[8] Cao Y., Zhang C., Shen W., Cheng Z., Yu L., Ping Q.: Poly( $N$-isopropylacrylamide)-chitosan as thermosensitive in situ gel-forming system for ocular drug delivery. Journal of Controlled Release, 120, 186-194 (2007).

DOI: 10.1016/j.jconrel.2007.05.009
[9] Geever L. M., Devine D. M., Nugent M. J. D., Kennedy J. E., Lyons J. G., Hanley A., Higginbotham C. L.: Lower critical solution temperature control and swelling behaviour of physically crosslinked thermosensitive copolymers based on $\mathrm{N}$-isopropylacrylamide. European Polymer Journal, 42, 2540-2548 (2006).

DOI: 10.1016/j.eurpolymj.2006.06.002

[10] Serres A., Baudyš M., Kim S. W.: Temperature and $\mathrm{pH}$-sensitive polymers for human calcitonin delivery. Pharmaceutical Research, 13, 196-201 (1996).

DOI: 10.1023/A:1016026711364

[11] Ramkissoon-Ganorkar C., Liu F., Baudyš M., Kim S. $\mathrm{W}$.: Modulating insulin-release profile from $\mathrm{pH} /$ thermosensitive polymeric beads through polymer molecular weight. Journal of Controlled Release, 59, 287298 (1999).

DOI: 10.1016/S0168-3659(99)00006-1

[12] Fundueanu G., Constantin M., Bortolotti F., Ascenzi P., Cortesi R., Menegatti E.: Preparation and characterisation of thermoresponsive poly[ $(N$-isopropylacrylamide-co-acrylamide-co-(hydroxyethyl acrylate)] microspheres as a matrix for the pulsed release of drugs. Macromolecular Bioscience, 5, 955-964 (2005).

DOI: 10.1002/mabi.200500099

[13] Varghese J. M., Ismail Y. A., Lee C. K., Shin K. M., Shin M. K., Kim S. I., So I., Kim S. J.: Thermoresponsive hydrogels based on poly( $N$-isopropylacrylamide)/ chondroitin sulfate. Sensors and Actuators B: Chemical, 135, 336-341 (2008).

DOI: $10.1016 /$ j.snb.2008.09.001

[14] Zhang J-T., Bhat R., Jandt K. D.: Temperature-sensitive PVA/PNIPAAm semi-IPN hydrogels with enhanced responsive properties. Acta Biomaterialia, 5, 488-497 (2009).

DOI: $10.1016 /$ j.actbio.2008.06.012

[15] Zhang J-T., Petersen S., Thunga M., Leipold E., Weidisch R., Liu X., Fahr A., Jandt K. D.: Micro-structured smart hydrogels with enhanced protein loading and release efficiency. Acta Biomaterialia, 6, 12971306 (2010).

DOI: $10.1016 /$ j.actbio.2009.11.005

[16] Zhao S. P., Cao M. J., Li L. Y., Xu W. L.: Synthesis and properties of biodegradable thermo- and $\mathrm{pH}$-sensitive poly[( $N$-isopropylacrylamide)-co-(methacrylic acid)] hydrogels. Polymer Degradation and Stability, 95, 719-724 (2010).

DOI: $10.1016 /$ j.polymdegradstab.2010.02.027

[17] Fundueanu G., Constantin M., Bortolotti F., Cortesi R., Ascenzi P., Menegatti E.: Poly[( $N$-isopropylacrylamide-co-acrylamide-co-(hydroxyethylmethacrylate))] thermoresponsive microspheres: An accurate method based on solute exclusion technique to determine the volume phase transition temperature. European Polymer Journal, 43, 3500-3509 (2007).

DOI: $\underline{10.1016 / j \text {.eurpolymj.2007.05.026 }}$ 
[18] Eeckman F., Moës A. J., Amighi K.: Synthesis and characterization of thermosensitive copolymers for oral controlled drug delivery. European Polymer Journal, 40, 873-881 (2004).

DOI: $10.1016 /$ j.eurpolymj.2003.11.010

[19] Eeckman F., Moës A. J., Amighi K.: Poly( $N$-isopropylacrylamide) copolymers for constant temperature controlled drug delivery. International Journal of Pharmaceutics, 273, 109-119 (2004).

DOI: $10.1016 /$ j.ijpharm.2003.12.013

[20] Schild H. G., Muthukumar M., Tirrell D. A.: Cononsolvency in mixed aqueous solutions of poly(N-isopropylacrylamide). Macromolecules, 24, 948-952 (1991).

DOI: $10.1021 / \mathrm{ma} 00004 \mathrm{a} 022$

[21] Schild H. G.: Poly( $N$-isopropylacrylamide): Experiment, theory and application. Progress in Polymer Science, 17, 163-249 (1992).

DOI: 10.1016/0079-6700(92)90023-R

[22] Constantin M., Fundueanu G., Bortolotti F., Cortesi R., Ascenzi P., Menegatti E.: Preparation and characterisation of poly(vinyl alcohol)/cyclodextrin microspheres as matrix for inclusion and separation of drugs. International Journal of Pharmaceutics, 285, 87-96 (2004).

DOI: $10.1016 /$ j.ijpharm.2004.07.025
[23] Otake K., Inomata H., Konno M., Saito S.: Thermal analysis of the volume phase transition with $\mathrm{N}$-isopropylacrylamide gels. Macromolecules, 23, 283-289 (1990).

DOI: $10.1021 / \mathrm{ma} 00203 \mathrm{a} 049$

[24] Katayama S., Hirokawa Y., Tanaka T.: Reentrant phase transition in acrylamide-derivative copolymer gels. Macromolecules, 17, 2641-2643 (1984).

DOI: $10.1021 / \mathrm{ma} 00142 \mathrm{a} 032$

[25] Tanaka T.: Collapse of gels and the critical endpoint. Physical Review Letters, 40, 820-823 (1978). DOI: $10.1103 /$ PhysRevLett.40.820

[26] Tokuyama H., Kato Y.: Preparation of poly( $N$-isopropylacrylamide) emulsion gels and their drug release behaviors. Colloids and Surfaces B: Biointerfaces, 67, 92-98 (2008).

DOI: $10.1016 /$ j.colsurfb.2008.08.003

[27] Wu J-Y., Liu S-Q., Heng P. W-S., Yang Y-Y.: Evaluating proteins release from, and their interactions with, thermosensitive poly ( $N$-isopropylacrylamide) hydrogels. Journal of Controlled Release, 102, 361-372 (2005).

DOI: $10.1016 /$ j.jconrel.2004.10.008 